\title{
Birth Weight Corrected for Gestational Age is Related to the Incidence of Down's Syndrome Pregnancies
}

\author{
Joris M. van Montfrans', Petra S.M. Bakker', Lyset T.M. Rekers-Mombarg², \\ Mirjam M. van Weissenbruch ${ }^{3}$, and Cornelis B. Lambalk' \\ ' Department of Obstetrics and Gynecology, Vrije Universiteit Medical Centre, The Netherlands \\ ${ }^{2}$ Department of Organisation, Management and Development, University of Twente, The Netherlands \\ ${ }^{3}$ Department of Pediatrics, Vrije Universiteit Medical Centre, The Netherlands
}

\begin{abstract}
Three recent studies reported that early depletion of the primordial follicle pool is likely to be an independent risk factor for Down's syndrome pregnancies. The size of the primordial follicle pool at birth is determined by oogenesis and by the rate of follicle atresia during the intra uterine period. Since intra uterine growth retardation was reported to be associated with a significantly reduced primordial follicle pool at birth, we investigated the possibility of a relation between low birth weight for gestational age and the risk of a Down's syndrome pregnancy. In a case control study, 95 women with a history of a Down's syndrome pregnancy and 85 controls provided information on their own birth weight and length of gestation. Birth weight standard deviation scores, indicating the difference in birth weight from a reference group, were significantly lower in Down's syndrome mothers than in controls. These findings illustrate that the risk of a Down's syndrome pregnancy is related to a low birth weight corrected for gestational age, possibly by a causal relation between intra uterine growth retardation and the size of the primordial follicle pool.
\end{abstract}

The specific risk factors for trisomy-21 pregnancies (causing Down's syndrome) are largely unknown (Eichenlaub-Ritter, 1996). Three recent studies however reported that early depletion of the number of primordial follicles in the ovaries is likely to be an independent risk factor for Down's syndrome (Freeman et al., 2000; Nasseri et al., 1999; van Montfrans et al., 1999). These studies report a significantly increased prevalence of signs of a depleted primordial follicle pool, irrespective of age, in women with a history of a trisomy-21 pregnancy and in women with other aneuploidies during pregnancy. Several factors other than maternal age may influence the size of the primordial follicle pool, and identifying these factors may provide more insight in the pathogenesis of Down's syndrome.

The formation of female germ cells occurs between the 2nd and 5th month of gestation (Crisp, 1992; Faddy et al., 1992). The maximum number of potential egg cells (approximately 4-6 million) is reached in the 5th month of gestation, after which stage this number starts to decline at an inverse exponential rate. At birth, around 600,000 primordial follicles remain and further decline occurs throughout childhood, puberty and the fertile life span, until approximately 1000 primordial follicles remain when menopause is reached.

The size of the primordial follicle pool at a given age is hypothesized to be influenced by both the maximum number of primary oocytes formed during the gestational period and the rate at which primordial follicles subsequently disappear. Since follicle formation and the majority of follicle atresia occur during the intra uterine period, the intra uterine environment may influence both processes. A study by De Bruin et al., (1998) reported that children born after intra uterine growth retardation (IUGR) had significantly reduced numbers of primordial follicles at birth, indicating that oocyte formation and / or oocyte atresia indeed may already be influenced during the intra uterine period.

Given the reported relation between IUGR and depletion of the primordial follicle pool at birth, we hypothesized that women born small for gestational age are at risk for Down's syndrome pregnancies in later life. We tested this hypothesis by assessing birth weight (corrected for gestational age) as indicator for IUGR in a group of women with a history of a Down's syndrome pregnancy and in a control group of women with children without Down's syndrome.

\section{Method}

The analysis was conducted in a previously published case control study into risk factors for a Down's syndrome pregnancy (van Montfrans et al., 1999). Cases were women with a history of a Down's syndrome pregnancy caused by trisomy-21 (confirmed by karyotyping), the control group consisted of women without a history of a Down's syndrome pregnancy and who had given birth to at least two live born children. Cases were recruited by advertisements in the magazine of the Dutch Down's Syndrome Foundation and through the patient files of the

Address for Correspondence: J.M. van Montfrans, Research Institute for Endocrinology, Reproduction and Metabolism, Department of Obstetrics and Gynaecology, Vrije Universiteit Medical Centre, P.O. Box 7057, 1007 MB Amsterdam, The Netherlands.E-mail: j.vanmontfrans@azvu.nl 
Department of Obstetrics and Gynecology of the Vrije Universiteit Medical Centre in Amsterdam, The Netherlands. Every Down's syndrome mother was asked to recruit one control participant from the same geographical area in The Netherlands and without blood relation to the Down's syndrome mother. Both cases and controls were aged under 41 years.

Data were acquired using a mailed questionnaire, addressing general health issues, gynaecological and obstetrical history and family history. Each woman was asked with an open question to report her own birth weight (as reported on the birth certificate) and length of gestational period. Data were entered into a database and analysed using SPSS Base 7.5 for Windows (SPSS Inc., Chicago, IL). Standard deviation (SD) scores for birth weight were calculated using the reference values by Usher and McLean (1969). The SD score for birth weight expresses the number of standard deviations of a subject's birth weight from the reference mean, adjusted for gestational age. The reference values by Usher and McLean (1969) were used since both the patient and control group in our study were born in the same time period as the women in this reference group. Continuous data were compared between the two groups using a Student's t-test. SD scores for birth weight were divided into categories and the number of patients per category was compared using a Chi-square test.

\section{Results}

We included 118 Down's syndrome mothers and 102 controls in the study. All participants completed and returned the questionnaire. Between the two groups, there were no significant differences in number of participants with chronic illnesses, current use of any medication, smoking

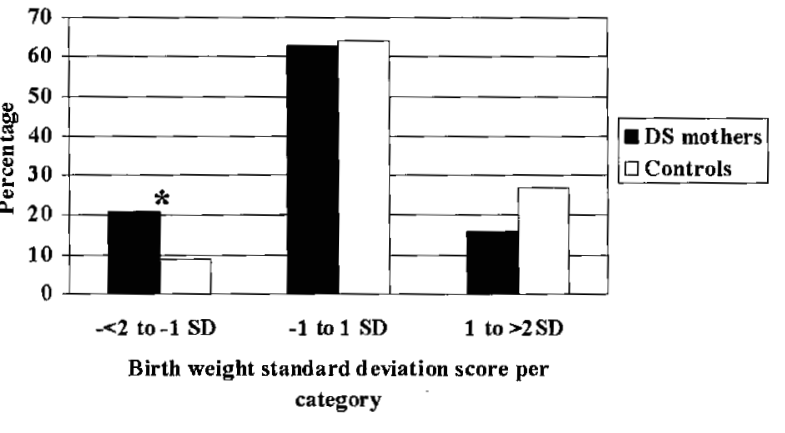

\section{Figure 1}

Standard deviation scores for birth weight per category in Down's syndrome mothers and controls.

Note: ${ }^{*} p<0.05$ representing a significantly increased number of Down's syndrome mothers with a standard deviation score for birth weight $<-1$ SD.

habits, alcohol consumption, parity or body mass index (data not shown).

Birth weight and gestational period were reported by 95 of 118 Down's syndrome mothers (81\%) and by 75 of 102 controls (77\%). Table 1 presents age, birth weight, length of the gestational period and SD scores for birth weight in the two groups. Mean SD scores for birth weight were significantly lower in Down's syndrome mothers than in controls $(p$ $=0.03$ ). The corresponding mean difference in birth weight adjusted for gestational age was 201 grams. Analysis of birth weight SD scores per category showed a significantly increased percentage of Down's syndrome mothers with a birth weight $<-1 S D$ from the mean birth weight (see Table 2 and Figure 1, birth weight $<-1 S D$ from the mean birth weight in $21 \%$ of the Down's syndrome mothers vs. $9 \%$ of the controls, respectively, $p<0.05)$.

\section{Table 1}

Age, Birth Weight, Length of Gestation and Standard Deviation Scores for Birth Weight in Down's Syndrome Mothers and Controls (Values are Mean $[S D]$ )

\begin{tabular}{lccc}
\hline Variable & Down's syndrome mothers $(n=95)$ & Controls $(n=75)$ & $95 \%$ Confidence interval \\
\hline Age (years) & $33.7(3.3)$ & $34.5(2.9)$ & -0.16 to 1.8 \\
Birth weight (grams) & $3413(527)$ & $3542(488)$ & -27 to 282 \\
Length of gestation (weeks) & $40.0(1.5)$ & $40.1(1.8)$ & -0.40 to 0.60 \\
Standard deviation score for birth weight & $0.0019(1.2)$ & $0.45(1.1)$ & 0.095 to 0.80 \\
\hline
\end{tabular}

\section{Table 2}

Birth Weight Standard Deviation (SD) Score Categories in Down's Syndrome Mothers and Controls and Chi-square Test for the Number of Women with a Birth Weight $S D$ Score $<-1 S D$ from the Mean: $p=0.038$.

\begin{tabular}{ccc}
\hline Birth weight SD score category & Down's syndrome mothers $(n=95)$ & Controls $(n=75)$ \\
\hline$<-2$ SD & $2(2 \%)$ & $1(1 \%)$ \\
-2 SD to -1 SD & $18(19 \%)$ & $6(8 \%)$ \\
-1 SD to 0 SD & $21(22 \%)$ & $15(20 \%)$ \\
S SD to 1 SD & $39(41 \%)$ & $33(44 \%)$ \\
1 SD to 2 SD & $10(11 \%)$ & $15(20 \%)$ \\
$>2$ SD & $5(5 \%)$ & $5(7 \%)$ \\
\hline
\end{tabular}




\section{$\overline{\text { Discussion }}$}

We found a significant relation between the occurrence of Down's syndrome pregnancies and low birth weight corrected for gestational age. This finding, taken together with the earlier reports of signs of a depleted oocyte reserve in women with a history of aneuploid pregnancies, supports the hypothesis of a relation between low birth weight corrected for gestational age and depletion of the primordial follicle pool, as reported by De Bruin et al. (1998).

Although standard deviation scores for birth weight were significantly lower in Down's syndrome mothers, the majority of these women was born after 37 weeks of gestation and had normal birth weights. This probably indicates that birth weight corrected for gestational age is not the only factor in the etiology of Down's syndrome pregnancies. However, it may be expected that relatively small differences in birth weight corrected for gestational age represent sub optimal intra uterine development. It is not known what mechanism causes the relation between reduced birth weight and the size of the primordial follicle pool at birth. De Bruin et al., (1998) proposed that fetal redistribution in case of placental insufficiency causes preferential blood flow to the vital organs and diminished blood flow towards other organs such as the ovaries, causing impaired follicle formation. Reduced birth weight may also be associated with accelerated follicle atresia or with structural changes in the circulatory system (impaired vascular elastine deposition; Martyn et al., 1998; Martyn \& Greenwald, 1997), possibly causing diminished blood flow to the ovaries and thereby causing follicle depletion. Alternatively, lower birth weight and an increased risk of aneuploid pregnancies may not be causally related, but epiphenomena of other disturbances in early fetal development, such as the possibility of abnormal genetic programming (Barker, 1997).

The data in this study were compared to the reference values of Usher and McLean (1969). We choose to use these values since the study subjects in their study were born in the same time period as the participants in our study. In order to avoid bias due to population differences between the Canadian population used by Usher and McLean (1969) and the Dutch population in our study, we compared both patients and controls to these reference values. Being a retrospective study based on a survey of women who volunteered from a publicity campaign and using data concerning gestational age and birth weight from different hospitals, the quality of the data in this study may be influenced by the abovementioned types of bias that are inevitable in this type of study. Further investigations need to be performed to confirm the results of this study. Ideally, these studies should include pedigree research of each woman to evaluate the effects of other known causes of differences in birth weight, such as environmental, nutritional and genetic factors.
In conclusion, we here report an association between reduced birth weight and Down's syndrome pregnancies, which may indicate that early depletion of the primordial follicle pool observed in women with a history of a Down's syndrome pregnancy is already established during intra uterine life.

\section{Acknowledgments}

The authors thank all participants and the Dutch Down's Syndrome Foundation for their cooperation. This study was supported by grant number 28-2770 of the Dutch Praeventiefonds, The Hague, The Netherlands.

\section{References}

Barker, D. J. (1997). The long-term outcome of retarded fetal growth. Clinical Obstetstrics and Gynecology, 40(4), 853-863.

Crisp, T. M. (1992). Organization of the ovarian follicle and events in its biology: Oogenesis, ovulation or atresia. Mutation Research, 296(1-2), 89-106.

De Bruin, J. P., Dorland, M., Bruinse, H. W., Spliet, W., Nikkels, P. G., \& te Velde, E. R. (1998). Fetal growth retardation as a cause of impaired ovarian development. Early Human Development, 51(1), 39-46.

Eichenlaub-Ritter, U. (1996). Parental age-related aneuploidy in human germ cells and offspring: A story of past and present. Environmental and Molecular Mutagenenesis, 28(3), 211-236.

Faddy, M. J., Gosden, R. G., Gougeon, A., Richardson, S. J., \& Nelson, J. F. (1992). Accelerated disappearance of ovarian follicles in mid-life: Implications for forecasting menopause. Human Reproduction, 7(10), 1342-1346.

Freeman, S. B., Yang, Q., Allran, K., Taft, L. F., \& Sherman, S. L. (2000). Women with a reduced ovarian complement may have an increased risk for a child with Down syndrome. American Journal of Human Genetics, 66(5), 1680-1683.

Martyn, C. N., Gale, C. R., Jespersen, S., \& Sherriff, S. B. (1998). Impaired fetal growth and atherosclerosis of carotid and peripheral arteries. Lancet, 352(9123), 173-178.

Martyn, C. N., \& Greenwald, S. E. (1997). Impaired synthesis of elastin in walls of aorta and large conduit arteries during early development as an initiating event in pathogenesis of systemic hypertension. Lancet, 350(9082), 953-955.

Nasseri, A., Mukherjee, T., Grifo, J. A., Noyes, N., Krey, L., \& Copperman, A. B. (1999). Elevated day 3 serum follicle stimulating hormone and/or estradiol may predict fetal aneuploidy. Fertility and Sterility, 71(4), 715-718.

Usher, R., \& McLean, F. (1969). Intrauterine growth of live-born Caucasian infants at sea level: Standards obtained from measurements in 7 dimensions of infants born between 25 and 44 weeks of gestation. Journal of Pediatrics, 74(6), 901-910.

Van Montfrans, J. M., Dorland, M., Oosterhuis, G. J., van Vugt, J. M., Rekers-Mombarg, L. T., \& Lambalk, C. B. (1999). Increased concentrations of follicle-stimulating hormone in mothers of children with Down's syndrome [letter]. Lancet, $353,1853-1854$. 